Ц итокиновый профиАь кожи у больных грибОвиАным МикОзОМ

(с) Карамова А.Э. ${ }^{*}$, Никоноров А.А., Вербенко Д.А., Знаменская Л.Ф., Воронцова А.А.

ФГБУ «Государственный научный центр дерматовенерологии и косметологии» Минздрава России 107076, Россия, г. Москва, ул. Короленко, д. 3, стр. 6

Цель исследования. Определить концентрации цитокинов в коже больных грибовидным микозом и оценить взаимосвязи со значением модифицированной шкалы оценки тяжести кожного поражения MSWAT.

Методы. С использованием технологии хМАР одновременно определяли концентрацию цитокинов: IL-1 $\beta$, IL-4, IL-6, IL-10, IL-17A, IL-17F, IL-21, IL-22, IL-23, IL-25, IL-31, IL-33, IFN- $\gamma$, sCD40L, TNF- $\alpha$ в биоптатах кожи 21 больного ранними (IA-IIA) стадиями грибовидного микоза и 4 здоровых лиц. Анализ и визуализация полученных данных проведены с использованием R Statistical Software for MacOS (версия 1.3.1056), свободная среда разработки программного обеспечения с открытым исходным кодом для языка программирования $\mathrm{R}$.

Результаты. Показано повышение уровня IL-4 и TNF- $\alpha$ в очагах поражения у больных ГМ по сравнению со здоровыми лицами ( $p=0,025$ и $p=0,012$ соответственно). С помощью корреляционного анализа выявлено фрормирование в коже больных ГМ цитокиновых сетей, не связанных со значением модифицированной шкалы оценки тяжести кожного поражения ISWAT. Обнаружена тенденция к отрицательной взаимосвязи шкалы $\operatorname{mSWAT}$ и IFN $\gamma(p=0,056)$.

Заключение. Ни один из цитокинов в очагах поражения и в визуально непораженной коже не был связан с величиной mSWAT.

Ключевые слова: грибовидный микоз, цитокины, интерлейкины, цитокины в коже.

Конфрликт интересов: авторы данной статьи подтвердили отсутствие конфрликта интересов, о котором необходимо сообщить.

Источник финансирования: исследование выполнено в рамках государственного задания ФГБУ «ГНЦДК» Минздрава России № 056-00015-18-00.

Для цитирования: Карамова А.Э., Никоноров А.А., Вербенко Д.А., Знаменская Л.Ф., Воронцова А.А. Цитокиновый профиль кожи у больных грибовидным микозом. Вестник дерматологии и венерологии. 2020;96(6):14-19. doi: https://doi.org/10.25208/vdv1202 


\title{
Skin cytokine profile in patients with mycosis fungoides
}

\author{
(C) Arfenya E. Karamova*, Alexandr A. Nikonorov, Dmitry A. Verbenko, Lyudmila F. Znamenskaya, Anastasiia A. Vorontsova
}

State Research Center of Dermatovenereology and Cosmetology, Ministry of Health of the Russian Federation Korolenko str., 3, bldg 6, Moscow, 107076, Russia

Aim of the study. To determine the concentration of cytokines in the skin of patients with mycosis fungoides and correlations between their concentration and the value of the modified scale for assessing the severity of skin lesions mSWAT.

Methods. The concentration of cytokines was simultaneously determined using XMAP technology: IL-1 $\beta$, IL-4, IL-6, IL-10, IL-17A, IL-17F, IL-21, IL-22, IL-23, IL-25, IL -31, IL-33, IFN- $\gamma$, sCD40L, TNF- $\alpha$ in skin biopsies of 21 patients with early (IA-IIA) stages of mycosis fungoides and 4 healthy individuals. Analysis and visualization of the obtained data were carried out using $R$ Statistical Software for MacOS (version 1.3.1056), a free open source software development environment for the $\mathrm{R}$ programming language. Results. An increase in the level of IL-4 and TNF- $\alpha$ in the lesions in patients with mycosis fungoides compared with healthy individuals was shown ( $p=0.025$ and $p=0.012$, respectively). Correlation analysis revealed the formation of cytokine networks in the skin of patients with mycosis fungoides that were not associated with the value of the modified scale for assessing the severity of skin lesions mSWAT. A tendency towards a negative relationship between the mSWAT and IFN $\gamma$ scales was found $(p=0.056)$. Conclusion. None of the cytokines in lesions and visually unaffected skin from patients with mycosis fungoides were associated with mSWAT values.

Keywords: cutaneous T-cell lymphoma, mycosis fungoides, cytokines, mSWAT, skin cytokine.

Conflict of interest: the authors of this article have declared the absence of conflicts of interest, which shall be reported if any.

Source of funding: the study was performed within the framework of the state assignment of the Federal State Budgetary Institution "State Scientific Center for Dermatovenerology and Cosmetology" of the Ministry of Health of Russian Federation No. 056-00015-18-00.

For citation: Karamova AE, Nikonorov AA, Verbenko DA, Znamenskaya LF, Vorontsova AA. Skin cytokine profile in patients with mycosis fungoides. Vestnik Dermatologii i Venerologii. 2020;96(6):14-19.

doi: https://doi.org/10.25208/vdv1202 
Введение

Грибовидный микоз - наиболее часто встречающееся заболевание из группы первичных Т-клеточных лимфом кожи, характеризующихся неконтролируемой пролиферацией малых и средних атипичных Т-лимфоцитов с формированием доминирующего клона в коже и по мере прогрессирования заболевания в лимфатических узлах, крови и внутренних органах [1, 2].

Злокачественная пролиферация Т-лимфоцитов при ГМ сопровождается изменением цитокинового профриля в коже больных: часть цитокинов интенсивно синтезируется и стимулирует пролиферацию атипичных клеток, в то время как синтез цитокинов - ингибиторов опухолевого роста угнетается, что способствует прогрессированию заболевания [3].

Цель исследования: определить концентрации цитокинов в коже больных грибовидным микозом и оценить взаимосвязи с клинической картиной заболевания.

\section{Методы}

Дизайн исследования

Одномоментное исследование.

Критерии соответствия

В исследование включали пациентов старше 18 лет с установленным диагнозом Т-клеточной лимфомы кожи - грибовидный микоз на ранних стадиях (IA - IIA), не получающих в момент включения системной иммуномодулирующей и/или иммуносупрессивной терапии.

\section{Условия проведения}

Исследование проводилось на базе Федерального государственного бюджетного учреждения «Государственный научный центр дерматовенерологии и косметологии» Министерства здравоохранения Российской Федерации.

\section{Продолжительность исследования}

В статье представлены результаты исследования, полученные в период с 2018 по 2020 г.

\section{Материал и методы}

В исследование последовательно включен 21 больной грибовидным микозом на ранних стадиях (IB-IIA) и 4 здоровых добровольца, которые подписали форму информированного согласия на участие в исследовании. Возраст пациентов варьировал от 28 до 74 лет (средний - 55,8 года). Средняя продолжительность заболевания составила 14,6 года. У 11 больных (52,3\%) была диагностирована стадия IB, у 10 больных $(47,7 \%)$ в связи с выявленным увеличением групп лимфатических узлов при проведении ультразвукового исследования - ІІА стадия. У всех больных присутствовали пятнистые и инфильтративно-бляшечные элементы, у 8 больных помимо пятен и бляшек наблюдались очаги пойкилодермии. Оценка тяжести поражения кожных покровов проводилась с применением клинического индекса оценки площади поражения BSA и модифицированной шкалы mSWAT $[4,5]$. Площадь поражения составляла от 8 до $64 \%$ (в среднем 26,4\%), значение модифицированной шкалы оценки тяжести кожного поражения mSWAT варьировало от 11 до 105 и в среднем составляло 39,9 балла.

У всех пациентов получен биоматериал из наиболее инфрильтрированного очага пораженной и участка визуально непораженной кожи.
Все полученные образцы взвешивались на лабораторных весах в промаркированных пробирках объемом 1,5 мл типа «Эппендорф» (Axygen), затем гомогенизировались с помощью автоматизированной системы для дезагрегации тканей Medimachine (Becton Dickinson, США) в специальных одноразовых камерах Medicons (Becton Dickinson, США) в течение 2 мин с добавлением 1 мл фросфратного буфрера $(\mathrm{pH}=7,4)$ к каждому образцу с последующей фильтрацией через одноразовый фрильтр с размером пор 50 мкм с целью предотвращения засорения проточной системы прибора.

Определение концентрации цитокинов в образцах проводилось на приборе Bio-Plex 200 (Bio-Rad) с использованием коммерческого набора реагентов Bio-Plex Pro Human Th17 Cytokine Panel 15-Plex (Bio-Rad), позволяющего одновременно определить содержание следующих цитокинов: IL-1 $\beta$, IL-4, IL-6, IL-10, IL-17A, IL-17F, IL-21, IL-22, IL-23, IL-25, IL-31, IL-33, IFN- $\gamma$, SCD40L, TNF- $\alpha$. Исследование каждого образца проводилось в дублях по 50 мкл суспензии согласно протоколу производителя с использованием автоматической станции отмывки микропланшетов Bio-Plex ProWash Station (Bio-Rad), шейкера для микропланшетов (Biosan). Для калибровки прибора использовали набор Calibrationkit (Bio-Rad). Учет результатов проводился автоматически в приложении Bio-PlexManager (BioRad) в размерности пг/мл. Полученные значения пересчитывали в соответствии с первоначальным весом биоптата и выражали в пг/мг.

Этическая экспертиза

Исследование проведено в соответствии с требованиями Декларации Хельсинки, протокол исследования одобрен локальным Этическим комитетом.

\section{Статистический анализ}

Принципы расчета размера выборки: размер выборки предварительно не рассчитывался.

Методы статистического анализа данных: анализ и визуализация полученных данных проведены с использованием R Statistical Software or MacOS (версия 1.3.1056), свободная среда разработки программного обеспечения с открытым исходным кодом для языка программирования R. Оценка распределения данных проводилась при помощи критерия Шапиро - Уилка. Данные представлены в виде медианы (25-75) методами описательной статистики (пакеты "skimr" и "psych"), для расчета корреляционных связей между выборками применялся корреляционный анализ по Спирмену с построением коррелограммы. Для оценки значимости различий между группами применяли критерий Краскела - Уоллиса с последующим апостериорным тестом Данна на множественность сравнений. Различия считались статистически значимыми при $p<0,05$.

\section{Результаты}

\section{Основные результаты исследования}

При оценке содержания цитокинов в коже здоровых лиц и в очагах поражения и видимо непораженной коже больных грибовидным микозом значимые различия выявлены только для IL-4 и TNF- $\alpha$ (рис. 1).

При проведении корреляционного анализа в неповрежденной коже (рис. 2) выявлена взаимосвязь между уровнями IL-6 и IFN- $\gamma(r=0,92)$, IL-17A $(r=0,65)$, IL-17F $(r=0,68)$ и IL-25 ( $r=0,5$, рис. 2). Уровень IFN- $\gamma$ был связан с IL-22 $(r=0,43)$, IL-17A $(r=0,66)$, IL-17F $(r=0,8)$, IL-25 $(r=0,69)$, IL-31 $(r=0,51)$. IL-22 - c IL-17A $(r=0,77)$, 

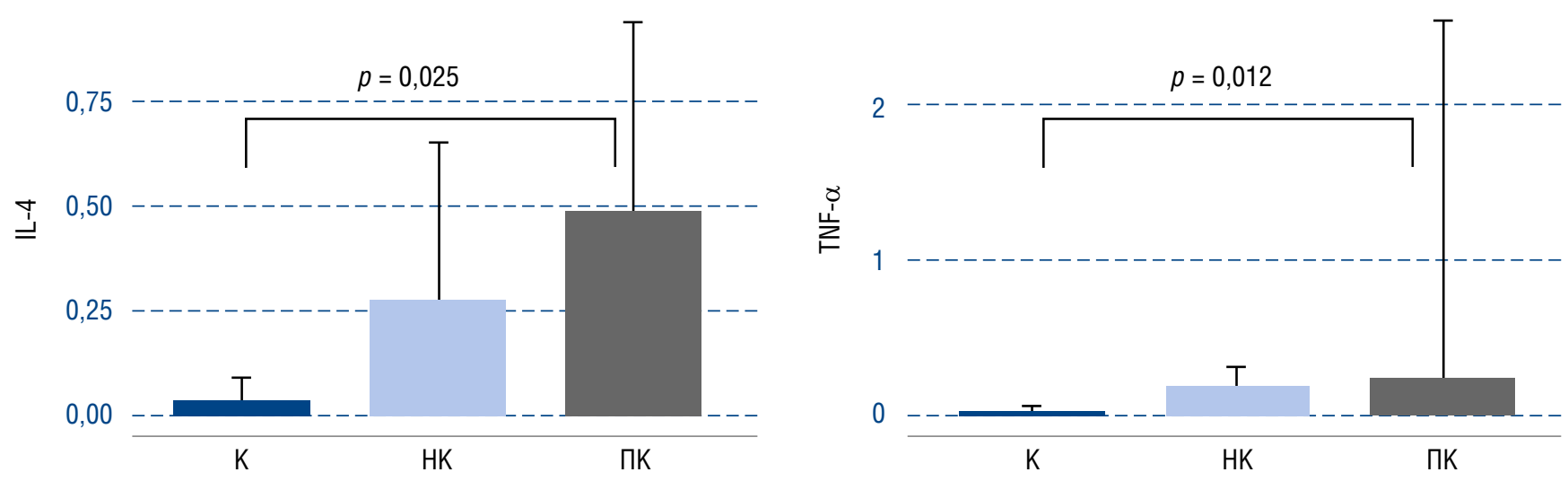

Рис. 1. Содержание IL-4 и TNF- $\alpha$ в коже здоровых лиц (К) и в визуально неповрежденной (НК) и поврежденной коже (ПК) пациентов с ГМ

Fig. 1. The concentration of IL-4 and TNF- $\alpha$ in the skin of healthy individuals (K), visually unaffected skin (HK) and lesions (ПK) of patients with mycosis fungoides

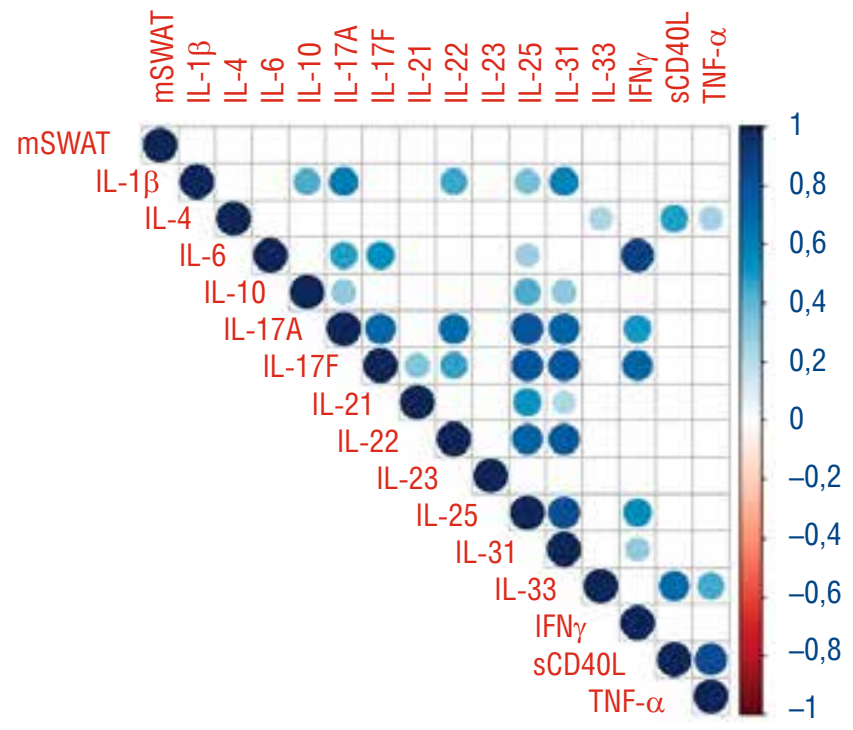

Рис. 2. Коррелограмма взаимосвязей цитокинов в неповрежденной коже (показаны только значимые корреляции, $p<0,05$ )

Fig. 2. Correlogram of cytokine relationships in visually unaffected skin (only significant correlations are shown, $p<0.05$ )

IL-17F $(r=0,64)$, IL-25 $(r=0,81)$, IL-31 $(r=0,83)$ и IL-1 $\beta$ $(r=0,63)$. IL-17A был связан с IL-25 $(r=0,79)$, IL-31 $(r=0,86), \mathrm{IL}-1 \beta(r=0,8), \mathrm{IL}-10(r=0,52)$. Уровень IL-17F был также связан с IL-25 $(r=0,86)$, IL-31 $(r=0,85)$, IL-1 $\beta$ $(r=0,42), \mathrm{IL}-10(r=0,43)$ и IL-21 $(r=0,54)$. II-25 - c IL-31 $(r=0,87), \mathrm{IL}-1 \beta(r=0,55), \mathrm{IL}-10(r=0,61)$. Уровни IL-31 C IL-1 $\beta(r=0,72)$, IL-10 $(r=0,52)$ и IL-21 $(r=0,46)$. IL-10 положительно коррелировал c IL-1 $(r=0,61)$, a IL-4 c IL-33 $(r=0,47)$, sCD40L $(r=0,65)$ и TNF- $\alpha(r=0,49)$. Была обнаружена связь уровня IL-33 c SCD40L $(r=0,77)$ и TNF- $\alpha(r=0,62)$. Высокий уровень корреляции был выявлен между SCD40L и TNF- $\alpha(r=0,89)$.

B пораженной коже (рис. 3) выявлено наличие положительной связи между уровнями IL-31 и IL-1 $\beta$ $(r=0,62)$. Уровень IL-31 был связан с уровнями TNF- $\alpha$ $(r=0,53)$, IL-17F $(r=0,63)$ и sCD40L $(r=0,63)$. IL-23 -

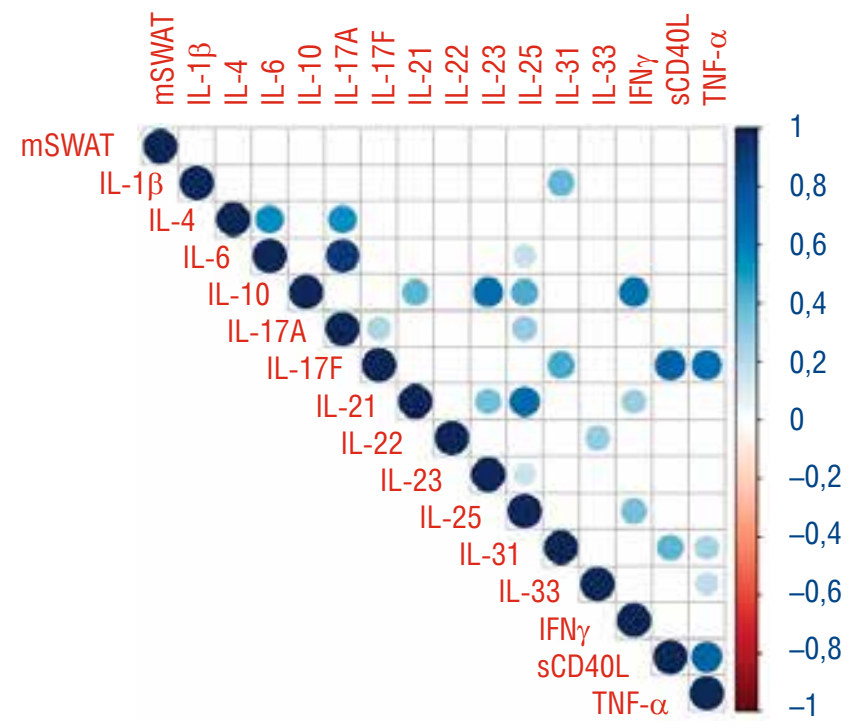

Рис. 3. Коррелограмма взаимосвязей цитокинов в пораженной коже (показаны только значимые корреляции, $p<0,05)$

Fig. 3. Correlogram of cytokine relationships in the lesions (only significant correlations are shown, $p<0.05$ )

c IL-21 $(r=0,4)$, IL-25 $(r=0,28)$, IL-10 $(r=0,69)$. IL-21 также коррелировал с IL-25 ( $r=0,74), \mathrm{IL}-10(r=0,35)$, IFN- $\gamma$ $(r=0,36)$. IL-25 - c IL-10 $(r=0,51)$, IFN- $\gamma(r=0,48)$, IL-6 $(r=0,54)$, IL-17A $(r=0,59)$. IL-10 - c IFN- $\gamma(r=0,71)$. IL-22 коррелировал c IL-33 $(r=0,53)$, а TNF- $\alpha-$ c IL-33 $(r=0,46)$, IL-17F $(r=0,79)$, sCD40L $(r=0,82)$. IL-17F был положительно связан с SCD40L $(r=0,82)$, IL-4 $(r=0,43)$ и IL-17A $(r=0,5)$. IL-4 был связан с уровнями IL-6 $(r=0,7)$ и IL-17A $(r=0,7)$. Очень тесная корреляция была выявлена между IL-6 и IL-17A $(r=0,95)$.

Корреляционный анализ концентрации цитокинов в пораженной коже и индекса mSWAT не позволил выявить статистически значимых различий, за исключением существенной тенденции к отрицательной взаимосвязи - чем выше индекс mSWAT, тем меньше IFN- $\gamma$ $(p=0,056)$; в неповрежденной коже статистически 
значимых различий выявлено не было. Важно отметить, что ни один из цитокинов в очагах поражения и визуально непораженной коже не был связан с индексом ISWAT.

Нежелательные явления

При проведении исследования нежелательных явлений отмечено не было.

\section{Обсуждение}

Продемонстрированное нами повышение содержания IL-4 в коже больных грибовидным микозом (ГМ) может свидетельствовать о значимой роли Th2, что, согласно литературным данным, в большей степени характеризует процессы, происходящие при прогрессировании заболевания в более поздние стадии [6]. Подавляющее действие IL-4 на экспрессию цитокинов Th1-типа (TNF- $\alpha$, IL-2, IL-12 и IFN- $\gamma$ ), преобладающих на ранних стадиях, стимулирует пролифрерацию злокачественных клеток [7]. В то же время нами выявлены различия для TNF- $\alpha$ в очагах поражения и видимо неповрежденной коже в сравнении с контрольной группой. Являясь центральным медиатором иммунных и воспалительных реакций, TNF- $\alpha$ может приводить к обострению заболевания у больных с пониженным уровнем экспрессии данного цитокина [8]. Доминирование на ранних стадиях Th1-клеток и повышенной экспрессии TNF- $\alpha$, IL-2, IL-12 и IFN- $\gamma$ может играть положительную роль в связи со способностью IFN- $\gamma$ ингибировать пролиферацию опухолевых клеток, тем самым снижая темпы прогрессирования заболевания, для которого характерны преобладание Th2 и экспрессия IL-4, IL-5, IL-10, IL-13, опосредованно ведущих к развитию иммуносупрессии и повышению чувствительности к бактериальным инфекциям [6].

Обнаруженные нами корреляционные взаимосвязи между уровнями цитокинов в коже больных грибовидным микозом свидетельствуют об участии в патологическом процессе Th1-цитокинов (TNF- $\alpha$, IL-2, IL-12 и IFN- $\gamma$ ), Th2 (IL-4, IL-5, IL-10, IL-13, IL-25, IL-31, IL-33), Th17 (IL-17A, IL-17F, TNF- $\alpha$, IL-6, IL-22) [9, 10], а также возможно и Th22-типа (IL-22, IL-10, TNF- $\alpha$ ) [11, 12]. Полученные результаты согласуются с литературными данными о роли, помимо Th1 и Th2, Th17-клеток [13]. T. Krejsgaard и соавт. (2013 г.) отметили высокий уро- вень экспрессии IL-17F в очагах поражения больных ГM [14]. Однако роль фенотипа Th17, и прежде всего IL-17, в патогенезе ГМ по-прежнему противоречива [15-17]. Известно, что продукция IL-22 связана с экспрессией IL-17A клетками фенотипа Th17, однако недавние исследования показали, что выработка IL-22 может происходить и при отсутствии IL-17A за счет экспрессии Th22-клетками [18, 19]. Т. Miyagaki и соавт. (2011 г.) обнаружили, что уровень экспрессии IL-22, IL-10, IL-4 в очагах поражения больных Т-клеточными лимфомами кожи значительно превышал уровень экспрессии IL-17A, IL-17F, IL-23p19, что, по мнению авторов, свидетельствует о более значимой роли IL-22 в прогрессировании заболевания по сравнению c IL-17 [20].

Выявленная нами тенденция к отрицательной взаимосвязи между индексом mSWAT и IFN- $\gamma$ согласуется с литературными данными о положительной роли IFN- $\gamma$ в течении заболевания [6].

Ограничения исследования

У всех больных грибовидным микозом, включенных в наше исследование, установлены стадии IB-IIA, однако степень выраженности клинических проявлений при одной и той же стадии различалась от ограниченных высыпаний с низкими значениями индекса mSWAT до распространенных с высокими значениями ISWAT. Несмотря на то что индекс mSWAT позволяет оценить тяжесть кожного поражения у больных ГМ, он не включен в систему стадирования, применяемую согласно рекомендациям Международного общества по лимфомам кожи (ISLE) и Европейской организации по изучению и лечению рака (EORTC), что не позволяет в полной мере оценить выраженность клинических проявлений и прогрессирование процесса [21].

\section{Заключение}

Проведенное нами исследование не выявило взаимосвязи между индексом MSWAT и определяемыми цитокинами. В то же время обнаружено существенное изменение взаимоотношений цитокинов в патологически измененной коже по сравнению с видимо непораженной, с фрормированием специфической цитокиновой сети.

\section{Литература/References}

1. Willemze R, Cerroni L, Kempf W, et al. The 2018 update of the WHO-EORTC classification for primary cutaneous Iymphomas. Blood.2019;133(16):1703-1714. doi:10.1182/blood-2018-11-881268

2. Jawed SI, Myskowski PL, Horwitz S, et al. Primary cutaneous T-cell lymphoma (mycosis fungoides and Sezary syndrome): part I. Diagnosis: clinical and histopathologic features and new molecular and biologic markers. J Am AcadDermatol 2014 Feb; 70(2):205; e1-16. PMID: 24438969 doi: 10.1016/j.jaad.2013.07.049

3. Matutes $\mathrm{E}$. The $2017 \mathrm{WHO}$ update on mature T- and natural killer (NK) cell neoplams. Int J LabHematol 2018 May;40(1):97-103. PMID: 29741263. doi:10.1111/ijlh.12817

4. Olsen EA, Whittaker S, Kim YH, et al. International Society for Cutaneous Lymphomas; United States Cutaneous Lymphoma Consortium; Cutaneous Lymphoma Task Force of the European Organisation for
Research and Treatment of Cancer. Clinical end points and response criteria in mycosis fungoides and Sézary syndrome: a consensus statement of the International Society for Cutaneous Lymphomas, the United States Cutaneous Lymphoma Consortium, and the Cutaneous Lymphoma Task Force of the European Organisation for Research and Treatment of Cancer. J Clin Oncol. 2011;29(18):2598-2607. doi:10.1200/JC0.2010.32.0630

5. Stevens SR, Ke MS, Parry EJ, et al. Quantifying skin disease burden in mycosis fungoides-type cutaneous T-cell lymphomas: The severity-weighted assessment tool (SWAT) Arch Dermatol. 2002;138: $42-48$.

6. Guenova E, Watanabe R, Teague JE, et al. TH2 cytokines from malignant cells suppress $\mathrm{TH} 1$ responses and enforce a global TH2 bias in leukemic cutaneous T-cell lymphoma. Clin Cancer Res 2013; 19:37553763. PMID: 23785046. doi:10.1158/1078-0432.CCR-12-3488 
7. Wolk K, Mitsui H, Witte K, et al. Deficient cutaneous antibacterial competence in cutaneous T-cell lymphomas: role of Th2-mediated biased Th17 function. Clin Cancer Res 2014; 20 (21):5507-16.PMID: 25212608. doi: 10.1158/1078-0432.CCR-14-0707

8. Amitay-Laish I, Guenova E, Ortiz-Romero PL, et al. The Course of Mycosis Fungoides under Cytokine Pathway Blockers: A Multicentre Analysis of Real-life Clinical Data. Acta Dermato-Venereologica. 2020 Sep;100(16):adv00277. doi: 10.2340/00015555-3642

9. Harrington LE, Mangan PR, Weaver CT. Expanding the effector CD4 Tcell repertoire: the Th17 lineage. Curr Opin Immunol 2006;18:349-56

10. Iwakura $Y$, Ishigame $H$. The IL-23/LL-17 axis in inflammation. $\mathrm{J}$ Clin Invest 2006;116:1218-22.

11. Duhen $T$, Geiger R, Jarrossay D, et al. Production of interleukin 22 but not interleukin 17 by a subset of human skinhoming memory $T$ cells. Nat Immunol 2009;10:857-63. 31

12. Eyerich S, Eyerich $K$, Pennino D, et al. Th22 cells represent a distinct human $T$ cell subset involved in epidermal immunity and remodeling. J Clin Invest 2009;119:3573-85.

13. Krejsgaard T, Ralfkiaer U, Clasen-Linde E, et al. Malignant cutaneous T-cell lymphoma cells express IL-17 utilizing the Jak3/ Stat3 signaling pathway. J Invest Dermatol 2011; 131(6):1331-8. PMID: 21346774. doi: 10.1038/jid.2011.27

14. Krejsgaard T, Litvinov IV, Wang $Y$, et al. Elucidating the role of interleukin-17F in cutaneous T-cell lymphoma. Blood 2013;122:943-950. PMID: 23801634. doi: 10.1182/blood-2013-01-480889
15. Yoo J, Shah F, Velangi S, et al. Secukinumab for treatment of psoriasis: does secukinumab precipitate or promote the presentation of cutaneous T-cell lymphoma? Clin Exp Dermatol 2019; 44: 414-417, 35-38.

16. Wolk K, Mitsui H, Witte K, et al. Deficient cutaneous antibacterial competence in cutaneous T-cell lymphomas: Role of Th2-mediated biased Th17 function. Clin Cancer Res 2014; 20; 5507-5516.

17. Miyagaki T, Sugaya M, Suga $H$, et al. IL-22, but Not IL-17, dominant environment in cutaneous T-cell lymphoma. Clin Cancer Res 2011; 17; 7529-7538.

18. Duhen T, Geiger R, Jarrossay D, et al. Production of interleukin 22 but not interleukin 17 by a subset of human skinhoming memory $T$ cells. Nat Immunol 2009;10:857-63. 31

19. Eyerich S, Eyerich K, Pennino D, et al. Th22 cells represent a distinct human $\mathrm{T}$ cell subset involved in epidermal immunity and remodeling. J Clin Invest 2009;119:3573-85.

20. Miyagaki T, Sugaya M, Suga $H$, et al. IL-22, but Not IL-17, Dominant Environment in Cutaneous T-cell Lymphoma. Clin Cancer Res 2011;17:7529-7538. Published OnlineFirst November 2, 2011.

21. Olsen E, Vonderheid E, Pimpinelli $N$, et al. Revisions to the staging and classification of mycosis fungoides and Sezary syndrome: A proposal of the International Sosiety for Cutaneous Lymphomas (ISCL) and the cutaneous lymphoma task force of the European Organization of Research and Treatment of Cancer (EORTC). Blood. 2007;110:1713-1722]. [The Modified Severity-Weighted Assessment Tool: A PASI/EASI System for Mycosis Fungoides FR-mSWAT: el "PASI/EASI" de la micosis fungoides A. Combalia, T. Estrach Actas Dermosifiliogr. 2018;109(8):745-746.

\section{Информация об авторах}

*Арфееня Эдуардовна Карамова — K.м.н.; адрес: Россия, 107076, г. Москва, ул. Короленко, д. 3, стр. 6; ORCID iD: http://orcid.org/0000-0003-3805-8489; eLibrary SPIN: 3604-6491; e-mail: karamova@cnikvi.ru

Александр Александрович Никоноров - д.м.H.; ORCID iD: http://orcid.org/ 0000-0001-7214-8176; eLibrary SPIN: 3859-7081; e-mail: nikonorov_all@mail.ru

Дмитрий Анатольевич Вербенко - к.б.н.; ORCID iD: http://orcid.org/0000-0002-1104-7694; eLibrary SPIN: 8261-6561; e-mail: verbenko@cnikvi.ru

Людмила Федоровна Знаменская - д.м.н.; ORCID iD: http://orcid.org/0000-0002-2553-0484; eLibrary SPIN: 9552-7850; e-mail: znam|@cnikvi.ru

Анастасия Александровна Воронцова - младший научный сотрудник; ORCID iD: http://orcid.org/0000-0002-3129-0050; eLibrary SPIN: 8334-2890; e-mail: vorontsova@cnikvi.ru

\footnotetext{
Information about the authors

*Arfenya E. Karamova — MD, Cand. Sci. (Med.); address: 3 bldg 6 Korolenko street, 107076, Moscow, Russia; ORCID iD: http://orcid.org/0000-0003-3805-8489; eLibrary SPIN: 3604-6491; e-mail: karamova@cnikvi.ru

Alexandr A. Nikonorov - MD, Dr. Sci. (Med.), Professor; ORCID iD: http://orcid.org/ 0000-0001-7214-8176; eLibrary SPIN: 3859-7081; e-mail: nikonorov_all@mail.ru

Dmitry A. Verbenko - Cand. Sci. (Biol.); ORCID iD: http://orcid.org/0000-0002-1104-7694; eLibrary SPIN: 8261-6561; e-mail: verbenko@cnikvi.ru

Lyudmila F. Znamenskaya - MD, Dr. Sci. (Med.); ORCID iD: http://orcid.org/0000-0002-2553-0484; eLibrary SPIN: 9552-7850; e-mail: znaml@cnikvi.ru

Anastasiia A. Vorontsova - junior research associate; ORCID iD: http://orcid.org/0000-0002-3129-0050; eLibrary SPIN: 8334-2890; e-mail: vorontsova@cnikvi.ru
}

Статья поступила в редакцию: 05.12.2020

Принята к публикации: 18.12.2020

Дата публикации: 30.12 .2020
Submitted: 05.12 .2020

Accepted: 18.12 .2020

Published: 30.12 .2020 